\title{
Markers umorali nella osteodistrofia uremica
}

\author{
G. Coen \\ Cattedra di Nefrologia e S.S. di Fisiopatologia Renale ed Ipertensione, Università \\ "La Sapienza” Roma
}

ella insufficienza renale cronica numerosi fattori, tra cui principalmente l'iperparatiroidismo secondario ed il difetto metabolico della vitamina $\mathrm{D}$, insieme ad altri quali l'acidosi metabolica, l'accumulo di alluminio ma anche l'età ed il sesso, contribuiscono allo sviluppo della malattia metabolica dell'osso nota come osteodistrofia renale. Le alterazioni scheletriche della insufficienza renale cronica insorgono piuttosto precocemente. Ad esempio già quando il filtrato glomerulare è dimezzato almeno il $50 \%$ dei soggetti presentano alcune alterazioni istologiche (1) ed il coinvolgimento del tessuto osseo risulta del tutto comune nei pazienti con insufficienza renale terminale, sia in fase predialitica che dialitica. Nel paziente con IRC in dialisi le lesioni vanno dall'iperparatiroidismo lieve, alle forme miste con associazione di iperparatiroidismo ed osteomalacia, alle forme di osteomalacia pura, specie negli stadi più avanzati del danno renale $(2,3)$, spesso associata $\mathrm{ad}$ acidosi metabolica più rilevante, mentre più raramente si riscontrano forme di iperparatiroidismo isolato grave. In aggiunta, negli ultimi anni, sono stati descritti quadri di patologia ossea adinamica (Adynamic Bone Disease degli autori anglosassoni), descritta inizialmente nei pazienti in dialisi (4), ma attualmente riscontrata anche nel paziente in fase conservativa della IRC (5). Dopo l'inizio della dialisi si assiste in generale ad un lento deterioramente della lesione ossea. Le classi istologiche di riferimento sono essenzialmente tre: una forma di iperparatiroidismo predominante, una forma di iperparatiroidismo misto a lesioni di tipo osteomalacico, con danno iperparatiroideo che varia da lieve a grave, e forme di malattia ossea osteodistrofica a basso turnover con o senza osteomalacia (6- 8). Queste forme venivano una volta considerate esclusivamente legate a tossicità da alluminio ed il termine identificante era Aluminum-Related Bone Disease (9). Attualmente con il venir meno della frequenza e della gravità della esposizione all'alluminio, si è messo in evidenza che la forma adinamica dell'osso, in assenza di sovraccarico di alluminio, è una forma di osteopatia molto frequente, con particolare riguardo ai pazienti con trattamento con calcio carbonato, con calcitriolo, oppure in terapia dialitica peritoneale $o$ affetti da diabete mellito e più tipicamente in età avanzata (10). Quindi la gamma dei tipi di lesione osteodistrofica è molto ampia e diversificata, ed a seconda del tipo di osteopatia può differire l'approccio terapeutico e la prognosi. Pertanto è spesso importante poter avere una diagnosi del tipo di osteopatia presente, informazione ben conseguibile mediante esame istologico su biopsia ossea.

Da molti anni l'interesse dei nefrologi e dei cultori delle malattie metabolitche dell'osso si sono rivolti ad identificare quei parametri umorali che possano essere di aiuto nella diagnosi non invasiva di osteopatia (11). I dosaggi umorali principali che hanno costituito fino ad oggi la base principale nel procedimento diagnostico, riguardano il PTH nelle sue varie forme, la fosfatasi alcalina, totale od isoenzima e l'osteocalcina o Bone GLA Protein (BGP) (12-14). Più recentemente si sono aggiunti altri marcatori che sono attualmente allo studio per valutarne il loro potenziale diagnostico. Il procollagene tipo 1 è il principale prodotto degli osteoblasti e viene utilizzato per costruire la struttura collagena dell'osteoide. Saggi radioimmunologici dei peptidi di estensione $\mathrm{N}$-terminale e $\mathrm{C}$ terminale della molecola del procollagene sono stati realizzati $(15,16)$ al fine di studiare in particolare il turnover dell'osso. Occorre tuttavia tenere presente che anche in altri tessuti si sintetizza sia pure in quantità minore il collagene tipo 1 per cui $\mathrm{i}$ frammenti del procollagene dosabili nel siero, possono avere una derivazione almeno parzialmente extraossea. Il propeptide carbossiterminale del procollagene tipo 1 (PICP) è una grossa molecola glicoproteica del peso molecolare di circa $100 \mathrm{kD}$, di struttura globulare. Scissa dalla molecola del procollageno una volta che questa è stata secreta dall'osteoblasta, questo frammento non viene incorporato nelle fibre collagene ed è liberato nei liquidi interstiziali e circolanti. Per il suo peso molecolare il 
TAB. I - ETÀ E PARAMETRI UMORALI NELLA IRC PREDIALISI

\begin{tabular}{lcc}
\hline & Pazienti & Valori Normali \\
\hline Età, anni & $54.1 \pm 15.8$ & - \\
Cr, mg/dl & $3.72 \pm 1.8$ & $0.5-1.1$ \\
PTH, C-term, ng/ml & $2.85 \pm 4.47$ & $0.2-0.9$ \\
PTH, intact, pg/ml & $150.6 \pm 172.7$ & $15-55$ \\
BGP, ng/ml & $26.9 \pm 19.2$ & $2-9$ \\
AP, $\mathrm{mU} / \mathrm{ml}$ & $88.4 \pm 38$ & $35-125$ \\
PICP, ng/ml & $166.9 \pm 61.9$ & $119 \pm 33$ \\
ICTP, ng/ml & $15.3 \pm 9.6$ & $1.7-5$ \\
\hline
\end{tabular}

peptide non può essere filtrato dal glomerulo, mentre viene captato dalle cellule endoteliali dei sinusoidi epatici dove esistono dei recettori specifici (17). Pertanto non risulta esservi un accumulo di PICP nel sangue circolante nella insufficienza renale cronica. Da studi in vitro ed in vivo $(18,19)$, la sintesi del procollagene tipo 1 da parte degli osteoblasti sembra essere inibita dal PTH. Tuttavia il PTH è in grado di stimolare la proliferazione dei preosteoblasti, cellule in grado di sintetizzare il procollagene tipo 1. Anche il calcitriolo esercita un effetto inibitorio sulla sintesi del procollagene tipo 1 (20). Parfitt et al (21) hanno riscontrato una stretta correlazione del PICP con i parametri istodinamici di formazione dell'osso in alcune condizioni di malattie metaboliche dell'osso. In aggiunta un aumento del PICP sierico è stato osservato a seguito di interventi chirurgici maggiori, come probabile risposta del tessuto connettivo nel processo di guarigione della ferita chirurgica (22).

L'importanza di disporre di una misura dell'entità del processo di riassorbimento dell'osso ottenibile in modo non invasivo ha suggerito la ricerca di sostanze umorali che potessero esprimere questo processo. Il collagene tipo 1 è una molecola complessa ad elice triplice costituita da due catene alfa 1 ed una alfa 2 . Nelle cisterne endoplasmatiche degli osteoblasti, ciascuna catena va incontro ad idrossilazione di circa 100 proline e 10 lisine ad idrossiprolina ed idrossilisina (23). L'idrossilisina viene glicosilata mediante agganciamento di galattosio o glucosiogalattosio dando luogo al glucosil-galattosil-idrossilisina. Dopo questi processi di idrossilazione le catene costitutive della molecola del procollagene si uniscono in modo stabile formando la molecola di procollagene tipo 1 , fenomeno che non permette nessuna ulteriore modificazione della molecola. Escreta dal-

TAB. II - CORRELAZIONI DEI PARAMETRI UMORALI ED ISTOMORFOMETRICI NELLA IRC PREDIALISI (valori di r)

\begin{tabular}{lcccc} 
& iPTH & BGP & AP & PICP \\
\hline Cr & $.576(63)$ & $.324(63)$ & $.368(64)$ & $.010(26)$ \\
ObS/BS & $.825(51)$ & $.529(63)$ & $.394(64)$ & $.217(26)$ \\
OcS/BS & $.626(51)$ & $.562(63)$ & $.699(64)$ & $.047(26)$ \\
& & & & \\
dLS/BS & $.349(39)$ & $.135(34)$ & $.153(38)$ & $.512(19)$ \\
MS/BS & $.084(39)$ & $-.157(34)$ & $-.044(38)$ & $.494(19)$ \\
MAR & $.053(39)$ & $.026(34)$ & $.384(38)$ & $.700(19)$ \\
BFR/BS & $.201(39)$ & $.048(34)$ & $-.012(38)$ & $.689(19)$ \\
AjAR & $-.130(39)$ & $-.139(34)$ & $-.168(38)$ & $.690(19)$ \\
\hline
\end{tabular}

l'osteoblasta e liberata dai peptidi di estensione, i residui lisinici della molecola vanno incontro ad ossidazione formando dei ponti tra catene e tra molecole, contenenti strutture piridiniche. Il processo di riassorbimento dell'osso può essere monitorizzato misurando l'escrezione urinaria (24) oppure i livelli plasmatici dei frammenti di collagene contenenti queste strutture piridiniche. Recentemente un metodo di dosaggio radioimmunologico del telopeptide aminoterminale del collagene tipo 1 (ICTP), contenente la struttura piridinica, è stato realizzato (25). Questo dosaggio è specifico del collagene dell'osso e può essere impiegato per il dosaggio nel siero, un rilievo molto importante per i pazienti in uremia terminale. L'ICTP è un peptide di piccole dimensioni, del peso approssimativo di $12 \mathrm{kD}$, normalmente filtrato dal glomerulo. Pertanto l'ICTP è parzialmente ritenuto nel siero nella insufficienza renale.

La nostra esperienza nei pazienti uremici riguarda sia lo stadio conservativo della insufficienza renale che quello dialitico. Nella insufficienza renale predialisi (26) lo studio è stato svolto in 68 pazienti, a vari stadi del danno renale, affetti da varie nefropatie croniche. Nessuno dei pazienti era sottoposto a trattamenti che potessero interferire con il metabolismo calcio-fosforico, né ricevevano vitamina $\mathrm{D}$ o analoghi. In tutti i pazienti veniva eseguita una biopsia ossea transiliaca, dopo doppia marcatura con tetraciclina, per la valutazione istomorfometrica statica e dinamica. Nella Tabella I sono riportati i valori medi dei vari parametri clinici ed umorali. Oltre agli aumenti osservati per i parametri iPTH, Fosfatasi Alcalina e BGP, si è riscontrato un aumento significativo del PICP, ma di dimensioni nettamente inferiori che per il iPTH. Un incremento relativamente maggiore era rilevabile per il parametro ICTP, parametro che risultava aumentato in misura corrispondente all'incremento del BGP. Pertanto è probabile che per ambedue questi parametri umorali operi, oltre che un aumento della produzione ossea, anche un fattore ritentivo legato alla riduzione della funzione renale, come d'altronde era atteso. Per quanto riguarda infatti la correlazione tra livelli di PICP e ICTP e livelli sierici di creatinina (Tab. II), si è osservato che la PICP non si correla con i livelli di cratinina, mentre vi è una correlazione significativa li- 


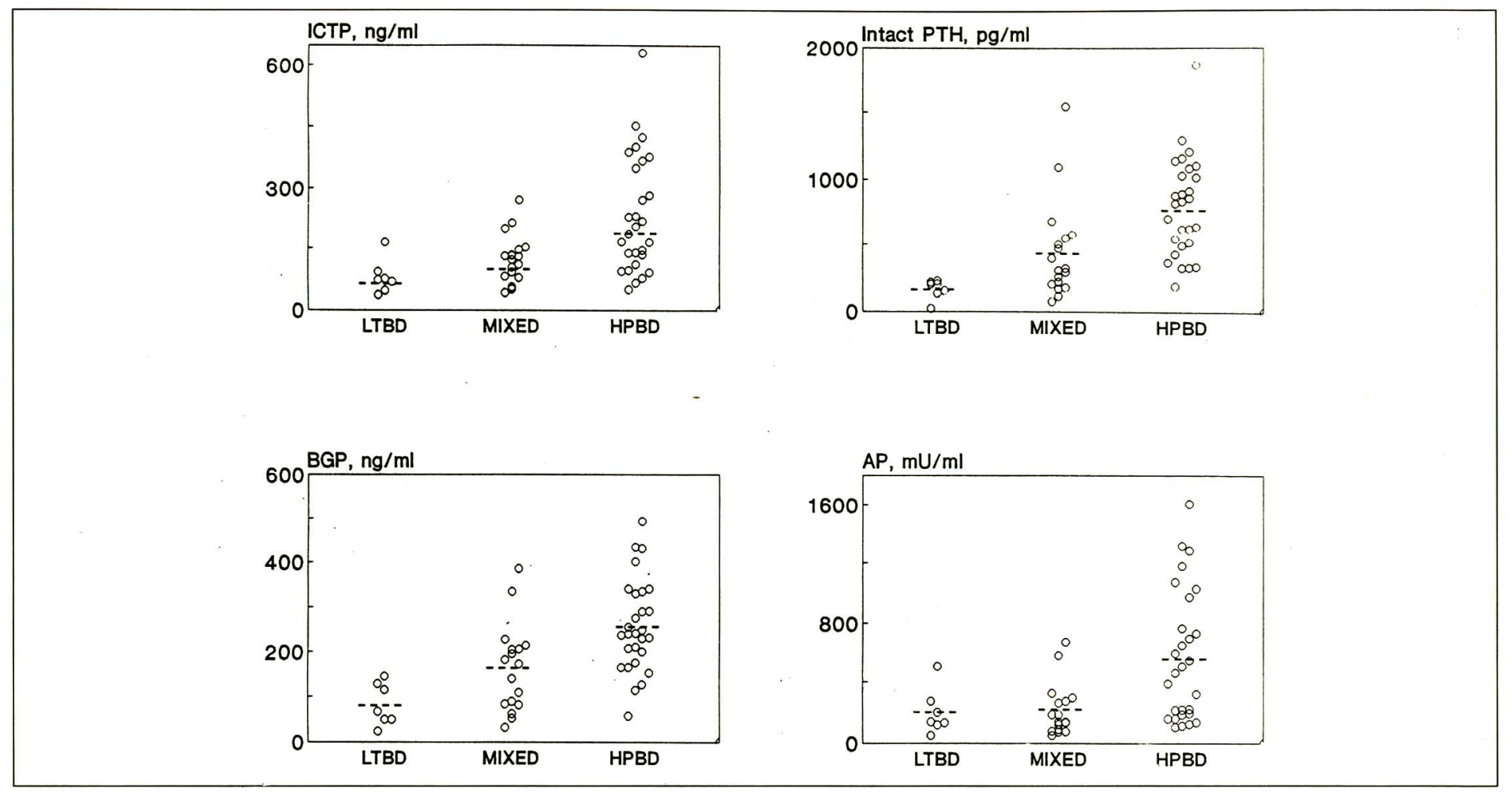

Fig. 1 - Valori di ICTP, PTH intatto, BGP e fosfatasi alcalina nei pazienti con osteodistrofia a basso turnover (LTBD), nelle forme miste (Mixed) e nella osteodistrofia a prevalente iperaparatiroidismo $(H P B D)$

neare tra ICTP e creatininemia. Evidentemente i livelli di PICP non risentono della riduzione del filtrato glomerulare in quanto condizionati dal metabolismo a sede epatica (17). I livelli sierici di ICTP si correlano in modo esponenziale con i valori della clearance della creatinina, così come i livelli di BGP (12), dimostrando bene il processo ritentivo legato all'insufficienza renale. L'analisi di correlazione lineare tra dati istomorfometrici statici e dinamici (Tab. II), eseguita per i parametri standard e per il PICP, ha dimostrato che questo parametro si correla significativamente con i principali indici di formazione dell'osso. Pertanto il PICP potrebbe essere un indice valido del processo osteoformativo almeno nella fase predialitica della insufficienza renale. Mancano ancora dati per quanto riguarda le relazioni tra ICTP e dati istomorfometrici in questo stadio della insufficienza renale:

L'importanza del PICP nella valutazione della malattia ossea del paziente in dialisi è stata studiata recentemente ed i risul-

\section{TAB. III - ETÀ, ETÀ DIALITICA E DATI UMORALI DI 58 PAZIENTI IN EMODIALISI $(\mathrm{M} \pm \mathrm{SD})$}

\begin{tabular}{lcc}
\hline & Pazienti & Valori normali \\
\hline Età anni & $48.2 \pm 13.2$ & - \\
Età dial., mesi & $97.7 \pm 51.3$ & - \\
PTH intatto, pg/ml & $606.5 \pm 434$ & $15-55$ \\
BGP, ng/ml & $192.9 \pm 117$ & $2-8$ \\
AP, $\mathrm{mU} / \mathrm{ml}$ & $410.3 \pm 396$ & $35-125$ \\
ICTP, ng/ml & $179.7 \pm 154$ & $1.7-5$ \\
PICP, ng/ml & $261.4 \pm 101$ & $70-150$ \\
P, mg/dl & $5.3 \pm 1.3$ & $3-4.5$ \\
\hline
\end{tabular}

tati ottenuti sono apparsi in parte contradditori. Hamdy et al. (27) hanno riscontrato una buona correlazione tra parametri osteoformativi e livelli di PICP in 18 pazienti in dialisi, parte dei quali presentavano sovraccarico scheletrico di alluminio. La nostra esperienza riguarda 58 pazienti in dialisi (28), tutti sottoposti a biopsia ossea transiliaca dopo doppia marcatura con tetraciclina. Nessuno dei pazienti, se si esclude uno, aveva un sovraccarico d'alluminio a livello di tossicità. Nessuno era in terapia con calcitriolo od analoghi della vitamina $D$. I valori medi per età, tempo in dialisi e dei parametri umorali sono riportati nella Tabella III. I valori medi del iPTH intatto e della fosfatasi alcalina erano nettamente aumentati rivelando mediamente un grado importante di iperparatiroidismo. I livelli sierici di PICP erano aumentati, ma nettamente meno del iPTH, della BGP e dell'ICTP. Questi ultimi due parametri erano in assoluto quelli con valori relativamente più elevati, certamente in rapporto oltre che alla entità della loro produzione ossea, anche alla ritenzione sierica dei peptidi, mal dializzabili e normalmente eliminati per filtrazione glomerulare. L'analisi di correlazione linea- 
TAB. IV - CORRELAZIONI TRA PARAMETRI UMORALI ED ISTOMORFOMETRICI IN 58 PAZIENTI IN EMODIALISI (valori di r)

\begin{tabular}{lccccc} 
& iPTH & BGP & AP & PICP & ICTP \\
\hline ObS/BS & .696 & .704 & .677 & .117 & .550 \\
OcS/BS & .549 & .598 & .566 & .141 & .478 \\
& & & & & \\
BFR/BS & .616 & .672 & .806 & .155 & .627 \\
AjAR & .563 & .642 & .674 & .194 & .508 \\
\hline
\end{tabular}

TAB. V - VALORI MEDI DEI PARAMETRI UMORALI ED ISTOLOGICI NEI PAZIENTI CON OSTEOPATIA A BASSO TURNOVER, OSTEOPATIA MISTA, ED IPERPARATIROIDISMO

\begin{tabular}{|c|c|c|c|}
\hline & $\begin{array}{l}\text { Basso-Turnover } \\
(\mathrm{n}=7)\end{array}$ & $\begin{array}{c}\text { Mista } \\
(\mathrm{n}=18)\end{array}$ & $\begin{array}{l}\text { Iperparatiroidismo } \\
(\mathrm{n}=28)\end{array}$ \\
\hline INT-iPTH (\$) & $157.7 \pm 69.7$ & $431.5 \pm 376.8$ & $798.0 \pm 378.3$ \\
\hline Cter-iPTH $(\$)$ & $6.7 \pm 6.0$ & $22.5 \pm 13.6$ & $43.1 \pm 22.8$ \\
\hline BGP $(\$)$ & $72.6 \pm 37.0$ & $161.4 \pm 94.3$ & $257.0 \pm 103.3$ \\
\hline $\mathrm{AP}(*)$ & $201.5 \pm 150.0$ & $206.8 \pm 173.8$ & $567.1 \pm 435.5$ \\
\hline PICP & $200.9 \pm 78.7$ & $258.6 \pm 93.5$ & $291.7 \pm 111.8$ \\
\hline $\operatorname{ICTP}(*)$ & $78.1 \pm 41.9$ & $123.0 \pm 59.3$ & $230.1 \pm 144.0$ \\
\hline OS/BS & $32.7 \pm 22.6$ & $38.3 \pm 12.9$ & $50.6 \pm 12.4$ \\
\hline $\mathrm{ObS} / \mathrm{BS}$ & $4.7 \pm 7.0$ & $7.9 \pm 4.7$ & $17.6 \pm 8.0$ \\
\hline ES/BS & $4.6 \pm 3.9$ & $6.6 \pm 2.4$ & $10.0 \pm 3.1$ \\
\hline $\mathrm{OcS} / \mathrm{BS}$ & $1.2 \pm 2.1$ & $2.2 \pm 0.9$ & $3.9 \pm 1.7$ \\
\hline OTh & $15.4 \pm 9.7$ & $15.8 \pm 4.2$ & $19.8 \pm 4.1$ \\
\hline BFR/BS & $0.05 \pm 0.03$ & $0.2 \pm 0.12$ & $0.6 \pm 0.4$ \\
\hline
\end{tabular}

ANOVA $(*) \mathrm{p}<0.01 ;(\$) \mathrm{p}<.00006$

re tra i parametri umorali ed alcuni dei più importanti parametri istomorfometrici statici e dinamici (Tab. IV), ha dimostrato che sia iPTH che BGP, AP and ICTP si correlano significativamente con i parametri istologici mentre il PICP non raggiunge livelli di significatività. Pertanto il dosaggio sierico del PICP, peptide indicativo di sintesi dell'osteoide, non sembra avere un ruolo di rilievo nella previsione non invasiva della gravità e del tipo di osteopatia metabolica. Risultati simili sono stati comunicati da Gerakis et al (29). I nostri dati sembrano inoltre confermati da una analisi della capacità predittiva degli indici umorali per le diverse classi istologiche osteodistrofiche. I pazienti sono stati divisi a seconda delle classi istologiche: osteopatia a basso turnover, osteodistrofia mista ed iperparatiroidismo prevalente. I dati relativi ai parametri umorali per i tre gruppi sono riportati nella Tabella V. L'analisi della varianza ha identificato una netta significatività della differenza tra $\mathrm{i}$ valori medi del iPTH, BGP ed ICTP, mentre le differenze tra gruppi istologici per la PICP risultavano non significative. Nondimeno la capacità discriminante le classi istologiche del dosaggio dell'ICTP è nettamente inferiore a quella dell'iPTH intatto (Fig. 1).
Per quanto riguarda la discrepanza tra valore predittivo dei processi osteoformativi in predialisi ed in dialisi del PICP, non è possibile fornire una spiegazione adeguata. È possibile che in dialisi, quando mediamente i valori del Bone Formation Rate sono molto elevati, al contrario che nella fase predialitica, non sussista più un accoppiamento tra processo di sintesi dell'osteoide espresso dai livelli di PICP ed il processo della sua calcificazione, di cui sono indici principali la Fosfatasi Alcalina e la BGP. Non è escluso che la capacità del fegato di depurare il siero del PICP si potenzi con l'aumentare dei suoi livelli sierici. Inoltre, in condizioni di grave iperparatiroidismo, come in dialisi, l'eccesso di iPTH circolante potrebbe svolgere un effetto soppressivo della sintesi di procollagene tipo 1 da parte degli osteoblasti, come già osservato in esperimenti in vitro ed in vivo $(18,19)$.

A questo proposito ulteriori informazioni sul significato dei markers umorali dell'osso derivano da un ulteriore studio eseguito nei pazienti in dialisi sottoposti a paratiroidectomia per iperparatiroidsmo secondario grave (30). A seguito della paratiroidectomia subtotale, con caduta immediata e profonda dei livelli di iPTH intatto, della calcemia a livelli nettamente ipocalcemici e della fosforemia, si osserva un andamento di lieve incremento temporaneo dei livelli di AP e di $\mathrm{BGP}$ che tendono poi a calare dopo circa un mese, mentre i livelli di PICP subiscono una netta salita con massimo a due settimane circa, con incrementi dell'ordine delle 4-6 volte i valori basali, con successiva lenta caduta. Al contrario i livelli sierici di ICTP scendono consensualmente ai livelli calcemici (31).

Pertanto è verosimile che la rimozione delle paratiroidi porti ad una derepressione della attività protidosintetica degli osteobalsti, specie per quanto riguarda il procollagene tipo 1 , ad ulteriore conferma degli effetti soppressivi dell'ormone sulla sintesi di questa proteina. $\mathrm{Al}$ contrario la caduta dei livelli del ICTP è espressione del ridursi della attività osteoclastica con la caduta dei livelli sierici del PTH. In aggiunta dati simili sono stati ottenuti in altre osservazioni concernenti il trattamento con boli di calcitriolo nell'iperparatiroidismo secondario del paziente in dialisi. La somministrazione del calcitriolo conduceva ad un transitorio aumento dei livelli del PICP, connes- 
so con la soppressione del iPTH, mentre l'ICTP mostrava una riduzione consensuale con l'andamento del iPTH.

I dati sono in accordo con l'ipotesi che la soppressione del iPTH conduce allo stesso tempo, sia pur transitoriamente, ad aumentata sintesi di procollagene tipo 1 e ad una ridotta attività osteoclastica di cui è espressione la liberazione di ICTP. In conclusione la diagnostica della osteodistrofia renale mediante metodi non invasivi si giova dell'utilizzo di alcuni nuovi markers di sintesi e di demolizione dell'osso.

Alcune indicazioni sul significato di questi markers e sul loro possibile utilizzo sono state qui riportate. Ulteriori studi saranno necessari per un miglior approfondimento.

Al momento attuale rimane di fondamentale importanza clinica l'uso del dosaggio del iPTH e degli altri markers dei quali l'esperienza rimane più consolidata, a cui sembra si possa aggiungere il dosaggio dell'ICTP, quale reale nuovo marcatore del processo demolitivo dell'osso.

\section{Ringraziamenti}

Le ricerche citate sono state finanziate con $i$ fondi del MURST e della Università "La Sapienza" di Roma.

\section{BIBLIOGRAFIA}

1. Malluche HH, Ritz E, Lange $\mathrm{HP}$, et al. Bone histology in incipient and advanced renal failure. Kidney Int 1976; 9: 355-62.

2. Dahl E, Nordal KP, Attramadal A, Hase J, Flatmark A. Renal osteodystrophy in predialysis patients wothout stainable bone aluminum. Acta Med Scand 1988; 224: 157-64.

3. Mora Palma FJ, Ellis HAS, Cook DB, et al. Osteomalacia in patients with chronic renal failure before dialysis or transplantation. Q J Med 1983; 207: 332-48.

4. Moriniere P, Cohen Solal M, Belbrik S, Sebert JL, Fournier A. Disappearance of aluminic bone disease in a long term asynmptomatic dialysis population restricting $\mathrm{Al}$ (OH) 3: emergence of an idiopathic adynamic bone disease. Nephron 1989; 56: 93-101.

5. Carvalho AB, Jorgetti Cendoroglo Neto M, Vieira JGH, Drueke T, Draibe SA, Ajzen H. High incidence of adynamic bone disease $(A B D)$ in chronic renal failure predialysis (CRF) patients. Nephrol Dial Trnasplant 1992; 7: 755 .

6. Evans RA, Flynn J, Dunstan CR, George CRP, McDonnell GD. Bone metabolism in chronic renal failure. Miner Electrolyte Metab 1992; 7: 207-18.

7. Sherrard DJ, Ott S, Malney N, Andress D, Coburn J. Renal osteodystrophy: classification, cause and treatment. In: Frame B, Potts JT, Jr eds. Clinical Disorders of Bone and Mineral Metabolism. Amsterdam: Excerpta Medica, 1983; 254-9.

8. Malluche HH, Faugère MC. Atlas of mineralized bone histology, Basel: Karger, 1986.

9. Smith AJ, Faugère MC, Abreo K, Fanti P, Julian P, Malluche HH.
Aluminum-related bone disease in mild and advanced renal failure: evidence for high prevalence and morbidity and studies on etiology and diagnosis. Am J Nephrol 1986; 6: $275-83$.

10. Fournier A, Morinière $P$, Cohen Solal ME, et al. Adynamic bone disease in uremia: may it be idiopathic? Is it an actual disease? Nephron 1991; 58: 1-12.

11. Coen G, Mazzaferro S. Bone metabolism and its assessment in renal failure. Nephron 1994; 67: 383401.

12. Coen G, Mazzaferro $\mathrm{S}$, Bonucci E, et al. Bone GLA protein in predialysis chronic renal failure: effects of 1,25 $(\mathrm{OH}) 2 \mathrm{D} 3$ administration in a long-term follow-up. Kidney Int 1985; 28: 783-90.

13. Mazzaferro S, Coen G, Ballanti $\mathrm{P}$, et al. Osteocalcin , iPTH, alkaline phosphatase and hand $\mathrm{x}$-ray scores as predictive indices of histomorphometric parameters in renal osteodystrophy. Nephron 1990; 56: 261-6.

14. Coen G, Mazzaferro S, Ballanti $P$, et al. Two-site immunoradiometric intact parathyroid hormone assay versus C-terminal parathyroid hormone in predicting osteodystrophic bone lesions in predialysis chronic renal failure. J Lab Clin Med 1993; 122: 103-9.

15. Taubman MB, Goldberg B, Sherr CJ. Radioimmunoassay for human procollagen. Science 1974; 186: $1115-7$

16. Melkko J, Niemi S, Risteli L, Risteli J. Radioimmunoassay for the carboxyterminal propeptide of human type procollagen. Clin Chem 1990; 36: 1328-32.

17. Smedrod B, Melkko J, Risteli L, Risteli J. Circulating C-terminal propeptide of type 1 procollagen is cleared mainly via the mannose receptor in liver endothelial cells. Biochem J 1990; 271: 345-50.

18. Partridge NC, Dickson CA, Kopp K, Teitelbaum SL, Crouch 
EC, Kahn AJ. Parathyroid hormone inhibits collagen synthesis at both ribonucleic acid and protein levels in rat osteogenic sarcoma cells. Mol Endocrinol 1989; 3: 232-9.

19. Simon LS, Slovik DM, Neer RM, Krane SM. Changes in serum levels of type I and type III procollagen extension peptides during infusion of human parathyroid hormone fragment (1-34). J Bone Miner Res 1988; 3: 241-6.

20. Rowe DW, Kram BE. Regulation of collagen synthesis in fetal rat cavaria by 1,25-dihydroxyvitamin D3. J Biol Chem 1982; 257 : 8009-15.

21. Parfitt AM, Simon LS, Villanueva AR, Krane SM. Procollagen type I carboxyterminal extension peptide in serum as a marker of collagen biosynthesis in bone: correlation with iliac bone formation rates and comparison with total alkaline phosphatase. J Bone Miner Res 1987; 2: 427-36.

22. Haukipuro K, Melkko J, Risteli L, Kairaluoma MI, Risteli J. Connective tissue response to major surgery and postoperative infection. Eur J Clin Invest 1992; 22: 330-40.

23. Prockop DJ, Kivirikko KI, Tuderman L, Guzman NA. The biosynthesis of collagen and its disorders. N Engl J Med 1979; 301: 1323.

24. Seibel MJ, Robins S, Bilezikian JP. Urinary pyridinium crosslinks of collagen: specific markers of bone resorption in metabolic bone disease. Trends Endocrinol Metab 1992; 3: 263-70.

25. Risteli J, Elomaa I, Niemi S, Novamo A, Risteli L. Radioimmunoassay for the pyridinoline crosslinked carboxyterminal telopeptide of type 1 collagne: a new marker of bone collagen degradation. Clin Chem 1993; 39: 635-40.

26. Coen G, Mazzaferro S, Ballanti $\mathrm{P}$, et al. Procollagen type $1 \mathrm{C}$-terminal extension peptide in predialysis chronic renal failure. Am J Nephrol 1992; 12: 246-51.
27. Hamdy NAT, Risteli J, Risteli L, et al. Serum type I procollagen peptide: a non-invasive index of bone formation in patients on haemodialysis ? Neprhol Dial Transplant 1994; 9: 511-6.

28. Coen G, Ballanti P, Mazzaferro S, Pasquali M, Bonucci E. Procollagen type $1 \mathrm{c}$-terminal extension peptide, PTH and 1,25 $(\mathrm{OH}) 2 \mathrm{D} 3$ in chronic renal failure. Bone 1993; 14: 415-20.

29. Gerakis A, Nicolou Ch, Konstantinidis S, Apostolou Th, Spantidakis VI, Billis A. Is serum procollagen carboxyterminal propetide a useful index for the diagnosis of renal osteodystrophy ? Nephrol Dial Transplant 1994; 9: 908.

30. Coen G, Mazzaferro S, DeAntoni E, et al. Procollagen type I C-terminal extension peptide serum levels following parathyroidectomy in hyperparathyroid patients. Am J Nephrol 1994; 14: 106-12.

31. Coen G, Mazzaferro S, Chicca S, Pasquali M, Sardella D. Decreased bone collagen breakdown and increased osteobalstic procollagen syntehsis after parathyroidectomy. EDTA-ERA Abstracts, September 1993; 217. 